\title{
El control del resultado en el gasto de los fondos públicos $(*)$
}

SUMARIO: I. CRITERIOS ECONOMICO-PRIVADOS DEL RESULTADO. II. CATEGORIAS DE LOS CONTROLES ADMINISTRATIVOS. III. EXIGENCIAS PUESTAS A LOS CONTROLES. IV. CONTROLES DE LOS RESULTADOS Y SU VALORACION. V. PLURALIDAD DE LOS METODOS DE CONTROL DEL RESULTADO.

\section{CRITERIOS ECONOMICO-PRIVADOS DEL RESULTADO}

Con el gasto de fondos privados el individuo persigue unos objetivos, cuyo éxito es valorado en función de la medida en que satisfacen sus necesidades, entendidas en el más amplio sentido. Las ganancias obtenidas, la seguridad, la satisfacción, la prestación de ayudas y el alcance de prestigio son algunas de las referencias que servirán de parámetro para esta evaluación. Al gasto corresponde generalmente una contraprestación considerada como equivalente, que puede ser verificada operacionalmente $o$, al menos, presumida.

Un inversor cuenta, por ejemplo, con unos intereses determinados y un empresario aspira con aumentar sus ventas (aunque sea en una cuantía no exactamente cifrable) como consecuencia de un gasto adicional efectuado en publicidad. Este último ejemplo nos muestra que el control del resultado del gasto de fondos tampoco es en el ámbito

(*) Publicado en la revista Schriften zur öffentbichen Vezwaliung und offentbichen wirtschaft: «Erfolgskontrolle bei der Vezausgabung öffentbicher mitteln», Tomo 91, Baden-Baden, 1986, pp. 13-17. El INAP y la Redacción de DOCUMENTACIÓN ADMINISTRATIVA desean dejar constancia de su agradecimiento al autor y editores, por la autorización concedida para la traducción al castellano y reproducción del presente trabajo. 
económico privado tan sencillo como suele suponerse; pero, en cualquier caso, existen ya numerosos estudios para determinar métodos de economía empresarial que proporcionen técnicas eficaces para alcanzar los objetivos empresariales.

Esto resultará, no obstante, especialmente complicado cuando no se da el principio de equivalencia, es decir, cuando no exista una contraprestación equivalente y, consiguientemente, no se pueda determinar con exactitud el nexo causal entre el gasto y el resultado. Como ejemplo valga aquí lo siguiente: es realmente muy difícil determinar en qué casos y hasta qué punto la instalación de cantinas y de locales sociales puede mejorar el clima de trabajo en una empresa o reducir el índice de absentismo. Lo que, en definitiva, sí que se puede precisar en una empresa es el resultado financiero de la utilización de sus recursos globales disponibles.

Del resultado económico de la empresa se puede deducir, además, el beneficio habido para el cliente que compra los productos según sus necesidades y preferencias. Con lo cual se puede decir que - bajo unas condiciones económicas, sociales y de política ambiental muy determinadas- el interés egoísta de las ofertas y demandas en régimen de concurrencia, fomenta al mismo tiempo el bienestar general. Pero no se ha podido llegar aún a ningún acuerdo sobre el contenido de este orden económico-social. Lo cual, a decir verdad, no puede sorprendernos, si se tiene en cuenta que la complejidad de las relaciones causaefecto no admite verificaciones unívocas del resultado para las diferentes medidas de orden político.

En conclusión: el gasto de fondos privados beneficia en primer término, y de la manera más diversa, al propio prestador, y por otro lado surge, a su vez, como efecto reflejo de los recursos empleados, otro resultado: el beneficio deseado por el destinatario. De esta ventaja recíproca resulta el intercambio en especies, remunerado o inmaterial.

\section{CATEGORIAS DE LOS CONTROLES ADMINISTRATIVOS}

Si se trata, sin embargo, del gasto de fondos públicos no suele regir el citado principio de equivalencia ni tan siquiera el nexo causal. Y ello porque en los supuestos de inversiones, prestaciones sociales, gastos de defensa, asignaciones financieras, subvenciones, etc. -ya sean de carácter estatal o local - no suele darse una contraprestación directamente imputable; sin perjuicio, claro es, de que, mediante tales gastos, lo que se persiga es la consecución de determinados objetivos políticos (sociales, estatales, económicos y otros de diversa índole). Habrá que ver, por tanto, si y hasta qué punto se han obtenido realmente los efectos buscados. Dada la importancia del gasto de las Corpora- 
ciones territoriales en todos los ámbitos vitales, el establecimiento de un sistema de gastos debe venir acompañado por un control de los resultados y, en su caso, de la supervisión de los objetivos obtenidos.

Estos controles no son nada nuevo y siempre se han llevado a cabo; piénsese, por ejemplo, en los controles administrativos previos, coetáneos y posteriores, en las comprobaciones de la corrección formal, material y de cálculo, en la supervisión de presupuestos y en el examen de cálculo y control financiero. Se controla por medio de técnicas de supervisión jurídica, técnica y de servicio, de derechos de cooperación y de intervención, de reservas de autorización, de la obligación de rendir cuentas y de la posibilidad de su liquidación. Con razón, el control es una parte esencial de la burocracia, pues en la mayoría de los casos no se puede prescindir de este tipo de controles, ya que sin ellos, como dicta la experiencia, muy pronto aparecerían irregularidades. Pero en este lugar no se va a discutir el problema de la justificación ni tampoco el de la eficacia de estos controles del resultado. Se trata aquí más bien de poner de relieve un defecto notorio de la mayor parte de los controles existentes, a saber: el que ordinariamente no enjuicien las medidas administrativas desde los criterios de sus resultados económicos.

\section{EXIGENCIAS PUESTAS A LOS CONTROLES}

Los teóricos del sistema, los economistas del bienestar, los técnicos presupuestarios y analistas de evaluaciones y hasta cultivadores de los «Property-Rights» y del Presupuesto de base cero han realizado inumerables esfuerzos para determinar los resultados de las actividades estatales (inclusive las comunales). La actual práctica administrativa y presupuestaria adolece de algunos aspectos negativos sometidos a una crítica unánime, a saber: el orientarse por el input, su visión aislada y su formalismo jurídico. Un ejemplo de ello puede ser el siguiente: no es lícito medir el cumplimiento de los objetivos por las partidas asignadas en el presupuesto para la policía urbana, sino que ha de tomarse como referencia el objetivo perseguido con estos medios, que se verificará en el descenso de daños humanos y materiales en los accidentes de tráfico. Para atender mejor al resultado habrá que adoptar globalmente medidas de carácter interministerial y contemplar, a su vez, la posibilidad de alternativas no convencionales. En el citado supuesto se podrían suprimir, quizás, los gastos adicionales para las fuerzas policiales, elevando por otro lado lás exigencias para obtener el carnet de conducir. O se podría gravar a los causantes de accidentes de tráfico con tasas destinadas a la financiación de la actividad policial. (Aunque como esto último es tan poco habitual, y para algunos incluso tan absurdo, ni siquiera se ha llegado a plantear seriamente, aduciendo los 
problemas que conllevaría su realización o consideraciones de un Estado de Derecho.) Y, aunque de vez en cuando se haya apuntado este tipo de observaciones, lo que realmente falta es un cálculo matemático y orientado.

La teoría y práctica empresariales disponen, a tal efecto, de un acervo de investigaciones y de experiencias nada desdeñables: un hecho que, por otro lado, ni la burocracia ministerial ni los demás sectores administrativos suelen tomar en consideración, especialmente porque se limitan a identificar el cálculo económico con un sistema de reglas con las que sólo se pueden determinar costes, ingresos y beneficios. Pero está demostrado que la fundamentación operacional de las decisiones empresariales va mucho más allá, abarcando factores no ponderables, programaciones heurísticas, escalas pluridimensionales, cálculos de óptimización y pronósticos, juegos de roles y de simulación, etc. Estos procedimientos cualitativos y cuantitativos son especialmente indicados para los controles ex-ante y ex-post y se basan en un traslado a la actividad administrativa adaptado especialmente a las condiciones características de ésta.

\section{CONTROLES DE LOS RESULTADOS Y SU VALORACION}

Dicho de manera muy simplificada, aquí se confronta el resultado de la actividad empresarial con el objetivo dispuesto. Para este método teleológico es esencial que se evalúen primero económicamente tanto los medios como los fines. Esto no puede ser interpretado en un sentido demasiado estricto, tal y como suele suceder, ya que se trata más bien de contrastar sólo en un comienzo la relación existente entre el input y el output con la disposición de los factores y su salida. Acto seguido estas relaciones se incluyen dentro del contexto global de la empresa, pasando a analizar las interdependencias horizontales y verticales junto con sus efectos externos, tanto los positivos y los negativos para el personal como para los terceros directamente afectados y (actualmente) también para el medio ambiente en general, incluyendo la naturaleza.

Si además de la producción de prestaciones también se abarcan los efectos de las daciones (en las diversas formas de la actividad administrativa), habrá que añadir a la evaluación empresarial también la económica. El siguiente paso para una mejor calificación cuantitativa y cualitativa sería la realización de una "monetarización» de los efectos, es decir, efectuar su valoración. Ello permitiría comparar los diferentes valores subsumidos ahora en un denominador común, ya que si se abandona el plano económico y se decide efectuar una valoración social global de los efectos mediatos e inmediatos comienzan a surgir 
toda una serie de problemas de determinación. Resulta aconsejable, por todo lo dicho, elaborar en primer lugar la rentabilidad empresarial, y luego la económica, antes de intentar fijar categorías de una eficacia extraeconómica, ya que ello resulta ser de muy difícil determinación. La humanización de los puestos de trabajo, la protección del medio ambiente, los balances sociales, la utilización errónea del capital, los objetivos estatales, los efectos externos y la maximación del bienestar general son una serie de conceptos que bastan para reconocer la importancia de una eficacia global, tanto de las actividades públicas como de las privadas.

Son varias las disciplinas en las que se plantean los problemas anteriormente nombrados así: además de la teoría de la empresa, sobre todo las ciencias financieras, y las regionales, la sociología y la politología. Cada una de estas disciplinas puede aportar elementos específicos al control del resultado del gasto; pero ello tendrá el riesgo de llegar a una excesiva complejidad, que lo haría fracasar. Tratando, sin embargo, de evitar este defecto, habrá que tender a abarcar y valorar los efectos de las diferentes medidas una por una, en vez de pretender alcanzar soluciones globales. En esta misma línea es recomendable un método paulatino con una dirección centrífuga, es decir, relacionar primero entre sí las prestaciones administrativas en el lugar de trabajo, en el departamento o sección y en la Administración correspondiente y ponerlas en relación con el consumo, para seguidamente estudiar los efectos de estas actividades respecto de otras Administraciones y finalmente respecto de los afectados que no pertenezcan a la Administración pública. Siempre que uno no se limite en este control del resultado a un análisis de las ventajas y desventajas habrá que fijar unas medidas básicas y unas escalas de valoración para una eventual estimación monetaria. Esta estandarización a la que acaba de aludirse viene siendo aplicada, desde hace tiempo, en el cálculo comercial (por ejemplo, el valor de la adquisición o principio del valor mínimo), en la inspección industrial (por ejemplo, las regulaciones sobre el ruido de la maquinaria), en el seguro social (por ejemplo, la reducción porcentual de la capacidad de trabajo), en la medicina (por ejemplo, la cuantía de la indemnización según el grado del daño producido), etcétera. ¿Por qué no habría de ser posible, entonces, elaborar una tabla de valores consensuados que nos permitan controlar el resultado del gasto de los fondos públicos, por lo menos en algunos sectores parciales? 


\section{PLURALIDAD DE LOS METODOS DE CONTROL DEL RESULTADO}

Los artículos siguientes tienen como objetivo una mayor concienciación de esta cuestión. Está claro y es sabido que no puede haber una fórmula o técnica única, la cual, una vez le hayan sido proporcionados los datos adecuados, estudie los problemas y nos proporcione las soluciones ansiadas. El control del resultado viene a ser, en definitiva, la toma de una posición crítica y ponderada y el estudio de una posible integración de una parte en el todo, así como la profundización en procesos políticos y finalmente el aprender a entender. Todo lo cual presupone un conocimiento de los objetivos políticos y administrativos, de las condiciones marco y de las técnicas y exige, además, un entendimiento interdisciplinar del método, así como un trabajo con medidas de coste y producción y categorías de eficacia (relación fines y medios) y la efectividad (relaciones de debe y haber). Hay que insistir, por todo ello, en estas cuestiones, tanto en los estudios de capacitación inicial como en los de perfeccionamiento profesional. El presente volumen ha de servir a tal efecto como instrumento de trabajo.

Traducción: Julia NIETO KOENIG 